\title{
Rapid deployment of virtual mind-body interventions during the COVID-19 outbreak: feasibility, acceptability, and implications for future care
}

\author{
Kelly M. Trevino ${ }^{1}$ - Nirupa Raghunathan ${ }^{1}$. Shelly Latte-Naor ${ }^{1} \cdot$ Fernanda C. G. Polubriaginof ${ }^{1} \cdot$ Claus Jensen $^{1}$. \\ Thomas M. Atkinson ${ }^{1}$ • Nicholas Emard ${ }^{1}$. Christina M. Seluzicki ${ }^{1}$ • Jamie S. Ostroff ${ }^{1}$ • Jun J. Mao ${ }^{1}$ (D)
}

Received: 22 July 2020 / Accepted: 4 September 2020 / Published online: 9 September 2020

(C) Springer-Verlag GmbH Germany, part of Springer Nature 2020

\begin{abstract}
Introduction COVID-19 increased stress levels while reducing access to mind-body services in patients with cancer. We describe the rapid deployment of remotely delivered mind-body services to people with cancer during COVID-19, rates of participation, and acceptability from patients' perspectives.

Methods Eligible participants were patients with cancer age $\geq 18$ years enrolled in a single academic cancer center's online patient portal. Interventions included mind-body group therapy sessions in fitness, meditation, yoga, dance, tai chi, and music delivered using Zoom video conferencing. Sessions were 30-45 min and led by an integrative medicine clinician. Following each session, participants were asked to complete a three-item questionnaire assessing (1) satisfaction with the class session, (2) reduction in stress/anxiety, and (3) likelihood of recommending the class to others. Patients could also provide comments in real-time using the Zoom chat function.

Results Among 5948 unique visits, the most frequently attended classes were fitness $(n=2513,42.2 \%)$ followed by meditation $(n=1176,19.8 \%)$ and yoga $(n=909,15.3 \%)$. Of these visits, $3902(65.6 \%)$ had an associated completed questionnaire. Across class types, a large majority of participants reported being extremely satisfied $(n=3733,95.7 \%)$, experiencing extreme reductions in anxiety/stress $(n=3268,83.8 \%)$, and being extremely likely to recommend the class to others $(n=3605,92.4 \%)$. Fitness had the highest endorsement among class types (all $p$ values $<0.001$ ). Themes from the chat responses included gratitude, expressions of helpfulness, and feelings of connection.

Conclusion High utilization of and satisfaction with these virtual mind-body services demonstrate the significant potential of remote delivery to facilitate patient access to services.
\end{abstract}

Keywords Fitness $\cdot$ Meditation $\cdot$ Yoga $\cdot$ Stress $\cdot$ Virtual $\cdot$ Cancer

\section{Introduction}

The Severe Acute Respiratory Syndrome Coronavirus 2 (SARS-CoV-2, COVID-19) is an unprecedented global pandemic. The threat of infection, news of overwhelmed healthcare institutions, and significant disruption of daily life are stressful [1]. People with cancer are particularly vulnerable to stress during this time due to pre-existing anxiety stemming

Jun J. Mao

maoj@mskcc.org

1 Memorial Sloan Kettering Cancer Center, New York, NY, USA from their symptom burden, treatment concerns, and fear of cancer progression or recurrence. This anxiety is compounded by higher susceptibility to infections, delays in diagnosis and treatment, and reduced access to support networks due to COVID-19 [2,3]. The uncertainty associated with cancer is compounded by the uncertainty associated with COVID-19 for both patients in active treatment and survivors [4, 5]. There is an urgent need to address the enormous psychological burden of COVID-19 on those affected by cancer while allowing for necessary social distancing $[4,6,7]$.

This paper describes the rapid deployment of remotely delivered mind-body services to people with cancer during COVID-19, participation rates, and acceptability from patients' perspectives. 


\section{Methods}

\section{Participants and procedures}

We developed and provided free virtual mind-body group therapy sessions (i.e., fitness, meditation, yoga, dance, tai chi, and music) for adult patients (ages $\geq 18$ years) using the Zoom video conferencing platform during COVID-19. We informed patients about sessions through the institution's patient portal, including a link to register. Each class lasted 30$45 \mathrm{~min}$; the sessions did not allow for participant audio or video to protect privacy. We collected de-identified data between April 1 and May 31, 2020, under an Institutional Review Board-approved protocol.

\section{Measures}

Clinicians tracked participant attendance in a Research Electronic Data Capture (REDCap) database [8]. Following each session, participants were asked to complete a three-item questionnaire on Zoom using a five-point Likert scale (range for questions 1 and 2: $1=$ not at all to $5=$ extremely, range for question 3: $1=$ extremely unlikely to $5=$ extremely likely) to evaluate (1) satisfaction with the session, (2) reduction in anxiety/stress due to class participation, and (3) likelihood of recommending the class to others. Participants could also provide feedback via the Zoom chat function.

\section{Statistical analysis}

We conducted descriptive analyses of attendance overall and by class type, and satisfaction overall and by type. Chi-square analyses were conducted to compare patient ratings across class type. Responses were dichotomized into "extremely" (response 5) versus not (responses 1-4) because ratings were skewed to "extremely." Data were analyzed using STATA 12 for Windows [9]. Qualitative data were collected from the Zoom chat and coded to identify themes.

\section{Results}

Attendance tracking recorded 5948 unique visits. The most frequently attended classes were fitness $(n=2513,42.2 \%)$, followed by meditation $(n=1176,19.8 \%)$, yoga $(n=909$, $15.3 \%)$, music $(n=513,8.6 \%)$, dance $(n=424,7.1 \%)$, and tai chi $(n=413,6.9 \%)$. The average attendance per class was highest for fitness (mean $=76.2$ participants), followed by tai chi (mean $=29.5$ participants), yoga (mean $=26.7$ participants), meditation (mean $=24.0$ participants), dance (mean = 17.0 participants), and music (mean $=14.3$ participants).

Approximately two-thirds of participants $(n=3902$, 65.6\%) completed the questionnaire. Across classes, the majority of the sample reported being "extremely satisfied" $(n=3733,95.7 \%)$. Satisfaction ratings were highest for fitness and music classes $\left(X^{2}(5, n=3902)=61.99, p<0.001\right.$; Fig. 1$)$. Participants also reported the classes reduced stress and anxiety, with $83.8 \%(n=3268)$ reporting extreme anxiety/stress reductions. Anxiety/stress reduction ratings were highest for music and fitness classes $\left(X^{2}(5, n=3902)=41.61, p<0.001\right)$. Among participants, $92.4 \%(n=3605)$ were "extremely likely" to recommend classes to others. Ratings were highest for fitness and music classes $\left(X^{2}(5, n=3902)=82.68, p<0.001\right)$.

The primary theme that emerged from live chat comments was gratitude. Participants noted exercises they found useful and appreciated the privacy and safety associated with practicing at home. Despite the remote delivery, participants also reported feelings of love and connection during classes.

\section{Discussion}

The high utilization and acceptability of virtual mindbody services demonstrate the significant potential of remote delivery to facilitate cancer patient access to integrative medicine. In the context of COVID-19, in-person participation was rapidly curtailed. However, numerous barriers to in-person participation exist in the normal course of cancer care including travel burden, institutional resources (e.g., space), symptom burden, demanding treatment schedules, and susceptibility to infection in immunecompromised patients [10]. Prior research on virtual delivery of mind-body programs supports the feasibility and benefits of these services but has focused on mindfulness interventions delivered individually through a website or app [11-13]. Our virtual classes overcome barriers to inperson delivery while providing a range of services (e.g., fitness, music). Interestingly, satisfaction was high even as privacy considerations necessitated a unidirectional experience. Future programs that allow participants to interact with clinicians and each other may also reduce loneliness and social isolation, important treatment targets during and after COVID-19 for patients and survivors $[4,5]$. Research examining the effectiveness and implementation of these classes will provide insight into their benefits and the factors that impact their adoption, reach, and sustainability.

Fitness classes were the most frequently attended and received the highest satisfaction ratings, followed by meditation classes. In the context of COVID-19 stay-at-home restrictions and gym closures, fitness classes may have addressed a significant unmet need for physical activity. Meditation may have provided an accessible strategy for managing the stress of COVID-19. However, all classes received very high satisfaction ratings with most participants endorsing the highest rating ("extremely"). 


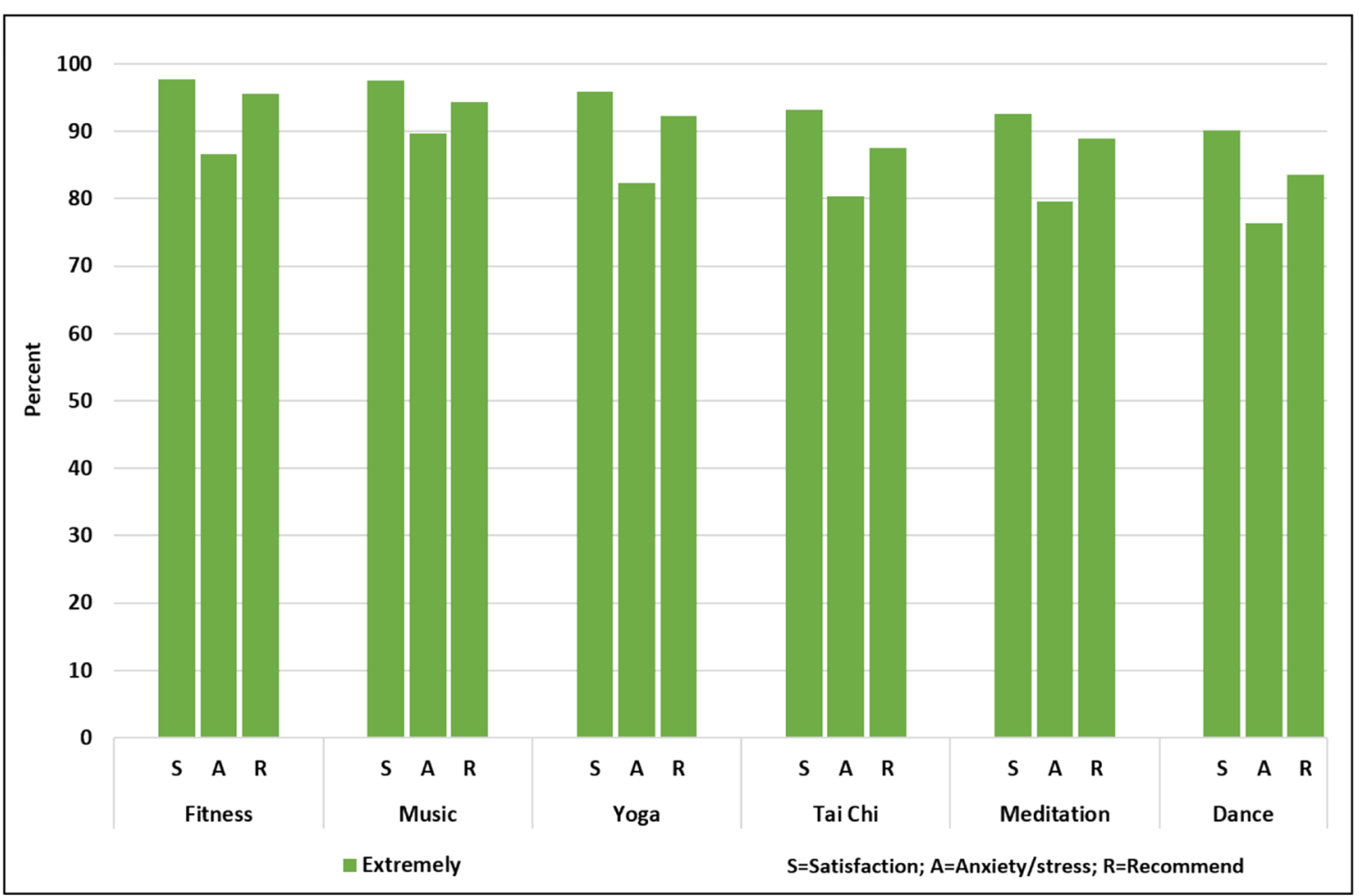

Fig. 1 Percentage of participants endorsing extreme satisfaction by modality

These mind-body services were provided at a single academic cancer center in an urban setting. To facilitate rapid provision, we did not collect potentially identifying information including patient sociodemographic characteristics. However, the patient population at this cancer center is largely white and highly educated. The feasibility of remote mindbody services for populations with less access to (e.g., rural locations) and familiarity with (e.g., older adults) online technology and identification of other barriers to online services (e.g., privacy concerns) are important areas of future research [5]. Furthermore, data on the number of unique patients who participated are not available and patients likely attended more than one session. Finally, classes were provided in the context of clinical care rather than a controlled research study and patients self-selected to participate. Research on the characteristics of patients who participate in online mind-body classes and reasons some patients do not participate will inform intervention modifications.

\section{Conclusion}

This initial examination of the feasibility and acceptability of virtual mind-body classes shows significant potential for remote delivery to improve patient access to these services. These results suggest that remote mind-body service delivery is acceptable to many patients with the potential to improve the wellbeing of a large proportion of people affected by cancer.

Acknowledgments We would like to thank the Integrative Medicine clinicians who conducted the classes as well as the administrative staff, all at Memorial Sloan Kettering Cancer Center, for their contributions to ensure that the mind-body program was successful. We would also like to thank the Patient and Family Advisory Council for Quality at Memorial Sloan Kettering Cancer Center for bringing the patient and caregiver perspective to the development and deployment of this program.

Author contributions All authors contributed to the study conception and design. Material preparation, data collection, and analysis were performed by Jun Mao, Kelly Trevino, Nirupa Raghunathan, Claus Jensen, Nicholas Emard, Fernanda Polubriaginof, and Christina Seluzicki. The first draft of the manuscript was written by Kelly Trevino and Nicholas Emard and all authors commented on previous versions of the manuscript. All authors read and approved the final manuscript.

Funding This manuscript is supported in part by a grant from the National Institutes of Health/National Cancer Institute Cancer Center (P30 CA008748) and the Translational and Integrative Medicine Research Fund at Memorial Sloan Kettering Cancer Center.

\section{Compliance with ethical standards}

Conflict of interest The author(s) declared the following potential conflicts of interest with respect to the research, authorship, and/or publication of this article: Dr. Mao reports grants from Tibet CheeZheng Tibetan Medicine Co. Ltd. and from Zhongke Health International LLC outside the submitted work. All other authors declare no potential conflicts of interest. 


\section{References}

1. Wang C, Pan R, Wan X, et al (2020) Immediate psychological responses and associated factors during the initial stage of the 2019 coronavirus disease (COVID-19) epidemic among the general population in China. Int J Environ Res Public Health 17

2. Linden W, Vodermaier A, Mackenzie R et al (2012) Anxiety and depression after cancer diagnosis: prevalence rates by cancer type, gender, and age. J Affect Disord 141:343-351

3. Ueda M, Martins R, Hendrie PC, et al (2020) Managing cancer care during the COVID-19 pandemic: agility and collaboration toward a common goal. J Natl Compr Canc Netw:1-4

4. Young AM, Ashbury FD, Schapira L, Scotté F, Ripamonti CI, Olver IN (2020) Uncertainty upon uncertainty: supportive care for cancer and COVID-19. Support Care Cancer 28:4001-4004

5. Chan A, Ashbury F, Fitch MI et al (2020) Cancer survivorship care during COVID-19-perspectives and recommendations from the MASCC survivorship study group. Support Care Cancer 28: 3485-3488

6. Hong YR, Lawrence J, Williams D Jr et al (2020) Population-level interest and telehealth capacity of US hospitals in response to COVID-19: cross-sectional analysis of Google search and national hospital survey data. JMIR Public Health Surveill 6:e18961

7. Kramer A, Kramer KZ (2020) The potential impact of the Covid-19 pandemic on occupational status, work from home, and occupational mobility. J Vocat Behav:103442
8. Harris PA, Taylor R, Thielke R, Payne J, Gonzalez N, Conde JG (2009) Research electronic data capture (REDCap)-a metadatadriven methodology and workflow process for providing translational research informatics support. J Biomed Inform 42:377-381

9. L.P. S: Stata statistical software: release 12.0. College Station, TX, StataCorp LP, 2011

10. Latte-Naor S, Mao JJ (2019) Putting integrative oncology into practice: concepts and approaches. 15:7-14

11. Cillessen L, Schellekens MPJ, Van de Ven MOM et al (2018) Consolidation and prediction of long-term treatment effect of group and online mindfulness-based cognitive therapy for distressed cancer patients. Acta Oncol 57:1293-1302

12. Russell L, Ugalde A, Orellana L, Milne D, Krishnasamy M, Chambers R, Austin DW, Livingston PM (2019) A pilot randomised controlled trial of an online mindfulness-based program for people diagnosed with melanoma. Support Care Cancer 27:2735-2746

13. Kubo A, Kurtovich E, McGinnis M et al (2019) A randomized controlled trial of mHealth mindfulness intervention for cancer patients and informal cancer caregivers: a feasibility study within an integrated health care delivery system. Integr Cancer Ther 18: 1534735419850634

Publisher's note Springer Nature remains neutral with regard to jurisdictional claims in published maps and institutional affiliations. 